\title{
Nonequilibrium quantum evolution of open systems*
}

\author{
Sang Pyo Kim \\ Department of Physics, Kunsan National University, \\ Kunsan 573-701, Korea
}

Received November 29, 1999

\begin{abstract}
We apply the Liouville-von Neumann (LvN) approach to open systems to describe the nonequilibrium quantum evolution. The Liouville-von Neumann approach is a unified method that can be applied to both time-independent (closed) and time-dependent (open) systems and to both equilibrium and nonequilibrium systems. We study the nonequilibrium quantum evolution of oscillator models for open boson and fermion systems
\end{abstract}

\section{Key words: Liouville-von Neumann approach, invariant operator, nonequilibrium quantum evolution, open boson system, open fermion system}

PACS: 03.65.-w, 05.30.-d

\section{Introduction}

The system of an open system interacts with an environment and depends on time implicitly or explicitly. A closed system can depend on time only when it is placed in an expanding Universe. It is the parametric interaction that forces the coupling constants of the system to depend on time. The time-dependent system cannot maintain the equilibrium state since the density operator defined in terms of the Hamiltonian does not satisfy the Liouville-von Neumann equation. Hence, the open system requires the nonequilibrium evolution through the interaction with the environment. Recently, the nonequilibrium evolution of open quantum systems has been an issue of much concern and debate [1].

There are two widely used methods to treat the nonequilibrium quantum systems. One is time-path integral method where path integrals are carried out over a contour of complex (imaginary) time to take into account the thermal nature of the system (for review and references, see [2]). The other is thermal field dynamics where the system of the open system interacts and makes a closed system with a fictitious system (for review and references, see [3]).

${ }^{*}$ This is an invited paper to the special issue on the problems of thermofield dynamics. 
It is the purpose of this paper to apply a recently developed field theoretical method, the so-called Liouville-von Neumann ( $\mathrm{LvN}$ ) approach to nonequilibrium quantum systems (for references, see [4]). It is a canonical method where the evolution of open systems is governed by the Tomonaga-Schwinger equation or by the functional Schrödinger equation. The $\mathrm{LvN}$ approach is well suited to describe the nonequilibrium quantum processes because the same LvN equation is used both to construct the Hilbert space of quantum states and to find the density operators. Also the method is nonperturbative in that the lowest approximation is the time-dependent Gaussian wave functional for a self-interacting system.

The organization of this paper is as follows. In section II we review the LvN approach and apply to a time-dependent oscillator. In section III we study three oscillator models for open boson systems and find their nonequilibrium evolution. In section IV we study open fermion systems motivated by a Dirac field interacting with an external source and a scalar field. In section $\mathrm{V}$ we conclude the paper.

\section{Liouville-von Neumann (LvN) approach}

The LvN approach is based on the idea by Lewis and Riesenfeld that the LvN equation can be used to find the exact quantum states of a time-dependent quantum system [5]. In particular, they solved exactly the time-dependent quantum oscillator in terms of an auxiliary equation. Recently, the author has found an oscillator representation, where the Fock space is constructed in terms of the classical solution [4,6-9]. Furthermore, the selection rule was put forth for the vacuum state for the time-dependent oscillator, which satisfies all criteria frequently imposed for well-known systems [9].

We elucidate the LvN approach to the nonequilibrium quantum evolution of open quantum systems. The LvN approach is based on two assumptions which are physically well-grounded. The one is that an open system should be described by a time-dependent quantum system obeying the Schrödinger equation $(\hbar=1)$

$$
\mathrm{i} \frac{\partial}{\partial t}|\Psi(t)\rangle=\hat{H}(t)|\Psi(t)\rangle .
$$

The other is that the statistical nature of an ensemble of the system should be described by the density operator satisfying the quantum LvN equation. In methodology, the $\mathrm{LvN}$ approach is based on the the following two theorems.

Theorem I. If $\hat{\mathcal{O}}(t)$ is a time-dependent invariant operator satisfying the quantum LvN equation

$$
\mathrm{i} \frac{d}{d t} \hat{\mathcal{O}}(t)=\mathrm{i} \frac{\partial}{\partial t} \hat{\mathcal{O}}(t)+[\hat{\mathcal{O}}(t), \hat{H}(t)]=0,
$$

then its eigenstates

$$
\hat{\mathcal{O}}(t)\left|\lambda_{n}, t\right\rangle=\lambda_{n}\left|\lambda_{n}, t\right\rangle
$$

provide the exact quantum state for equation (1) by

$$
|\Psi(t)\rangle=\sum_{n} c_{n} \exp \left[\mathrm{i} \int \mathrm{d} t\left\langle\lambda_{n}, t\left|\mathrm{i} \frac{\partial}{\partial t}-\hat{H}(t)\right| \lambda_{n}, t\right\rangle\right]\left|\lambda_{n}, t\right\rangle .
$$


This theorem was first proved by Lewis and Riesenfeld. In order to prove the theorem we use equations (1) and (2) to get the identity

$$
\left(\hat{\mathcal{O}}(t)-\lambda_{n} \hat{I}\right)\left[\left(\mathrm{i} \frac{\partial}{\partial t}-\hat{H}(t)\right)\left|\lambda_{n}, t\right\rangle\right]=0
$$

Then equation (5) implies that

$$
\left(\mathrm{i} \frac{\partial}{\partial t}-\hat{H}(t)\right)\left|\lambda_{n}, t\right\rangle=\alpha_{n}(t)\left|\lambda_{n}, t\right\rangle
$$

Theorem II. If $\hat{\mathcal{O}}_{1}(t)$ and $\hat{\mathcal{O}}_{2}(t)$ satisfy the LvN equation $(2)$, then $\hat{\mathcal{O}}_{1}(t) \hat{\mathcal{O}}_{2}(t)$ satisfies the same LvN equation. This follows from the identity

$$
\begin{aligned}
& \mathrm{i} \frac{\partial}{\partial t}\left(\hat{\mathcal{O}}_{1}(t) \hat{\mathcal{O}}_{2}(t)\right)+\left[\left(\hat{\mathcal{O}}_{1}(t) \hat{\mathcal{O}}_{2}(t)\right), \hat{H}(t)\right]= \\
& \quad=\left\{\mathrm{i} \frac{\partial}{\partial t} \hat{\mathcal{O}}_{1}(t)+\left[\hat{\mathcal{O}}_{1}(t), \hat{H}(t)\right]\right\} \hat{\mathcal{O}}_{2}(t)+\hat{\mathcal{O}}_{1}(t)\left\{\mathrm{i} \frac{\partial}{\partial t} \hat{\mathcal{O}}_{2}(t)+\left[\hat{\mathcal{O}}_{2}(t), \hat{H}(t)\right]\right\}=0 .
\end{aligned}
$$

In general, one can show that $F[\hat{\mathcal{O}}(t)]$ satisfies the $\operatorname{LvN}$ equation, if it is an analytical functional of $\hat{\mathcal{O}}(t)$ and $\hat{\mathcal{O}}(t)$ satisfies the $\mathrm{LvN}$ equation.

A few remarks should be made. Firstly, the LvN approach can also be applied to time-independent systems without any modification. In this case one can choose $\hat{\mathcal{O}}=\hat{H}$ and the energy eigenstates are the exact quantum states as expected from quantum mechanics. Secondly, according to the theorem II any invariant operator $\hat{\mathcal{O}}(t)$ can be used to define a density operator

$$
\hat{\rho}_{\mathcal{O}}(t)=\frac{1}{\operatorname{Tr}\left(\mathrm{e}^{-\beta \hat{\mathcal{O}}(t)}\right)} \mathrm{e}^{-\beta \hat{\mathcal{O}}(t)} .
$$

To describe the initial thermal equilibrium state a particular kind of invariant operator will be selected. In this sense the LvN approach can be applied to the nonequilibrium quantum evolution of time-dependent systems. Finally, the LvN approach can be extended to quantum fields the coupling constants of which depend on time. When a field is appropriately mode-decomposed, the system of the quantum field becomes an infinite system of finite-dimensional quantum mechanical systems.

We now turn to the most typical system of time-dependent oscillators. These systems occur in all areas of physics and play a prominent role partly because any system around an extremum can be approximated by a system of oscillators and partly because the classical and quantum evolution can be found exactly. Even a free field can be treated as a system of infinite number of oscillators via modedecomposition. Thus the study of a time-dependent oscillator will shed light on the nonequilibrium quantum evolution of a generic time-dependent quantum system.

Without loss of generality, we consider an oscillator the mass and frequency of which depend on time explicitly:

$$
\hat{H}(t)=\frac{1}{2 m(t)} \hat{p}^{2}+\frac{m(t)}{2} \omega^{2}(t) \hat{q}^{2} .
$$


In $[8,9]$ a pair of invariant operators are found

$$
\hat{A}(t)=\mathrm{i}\left(u^{*}(t) \hat{p}-m(t) \dot{u}^{*}(t) \hat{q}\right), \quad \hat{A}^{\dagger}(t)=-\mathrm{i}(u(t) \hat{p}-m(t) \dot{u}(t) \hat{q}),
$$

where $u$ satisfies the classical equation of motion

$$
\ddot{u}(t)+\frac{\dot{m}(t)}{m(t)} \dot{u}(t)+\omega^{2}(t) u(t)=0 .
$$

These invariant operators can be made the annihilation and creation operators by imposing the standard commutation relation for all times

$$
\left[\hat{A}(t), \hat{A}^{\dagger}(t)\right]=1
$$

which is guaranteed by the Wronskian condition

$$
m(t)\left(\dot{u}^{*}(t) u(t)-\dot{u}(t) u^{*}(t)\right)=\mathrm{i} .
$$

From the theorem II we can find a particularly simple functional of two invariant operators (10) called the number operator

$$
\hat{N}(t)=\hat{A}^{\dagger}(t) \hat{A}(t) .
$$

The vacuum state and the number states are defined by

$$
\hat{A}(t)|0, t\rangle=0, \quad|n, t\rangle=\frac{1}{\sqrt{n !}}\left(\hat{A}^{\dagger}(t)\right)^{n}|0, t\rangle .
$$

According to the theorem I the exact quantum state (4) is a linear superposition of number states. The density operator is defined by

$$
\hat{\rho}(t)=\frac{1}{Z} \mathrm{e}^{-\beta \omega_{0} \hat{N}(t)},
$$

where $Z$ is the partition function

$$
\begin{aligned}
Z & =\sum_{n=0}^{\infty}\left\langle n, t\left|\mathrm{e}^{-\beta \omega_{0} \hat{N}(t)}\right| n, t\right\rangle \\
& =\frac{1}{1-\mathrm{e}^{-\beta \omega_{0}}} .
\end{aligned}
$$

The density operator has got the coordinate representation

$$
\begin{aligned}
\rho\left(x, x^{\prime}, t\right) & =\frac{1}{Z} \sum_{n, n^{\prime}=0}^{\infty}\langle x \mid n, t\rangle\left\langle n, t\left|\mathrm{e}^{-\beta \omega_{0} \hat{A}^{\dagger}(t) \hat{A}(t)}\right| n^{\prime}, t\right\rangle\left\langle n^{\prime}, t \mid x\right\rangle \\
& =\frac{1}{Z} \sum_{n=0}^{\infty} \Psi_{n}(x, t) \Psi_{n}^{*}\left(x^{\prime}, t\right) \mathrm{e}^{-\beta \omega_{0} n},
\end{aligned}
$$

where

$$
\Psi_{n}(x, t)=\langle x \mid n, t\rangle
$$

are the harmonic oscillator wave functions. 


\section{Open boson system}

In this section we shall consider three oscillator models for open boson systems. These systems are motivated by a scalar field in a linear or quadratic interaction with an environment. The first two models have all the essential characteristics of a scalar field having a quadratic interaction with an external source

$$
\mathcal{L}=\frac{1}{2} \partial_{\mu} \phi(\mathbf{x}, t) \partial^{\mu} \phi(\mathbf{x}, t)-\frac{m_{0}^{2}}{2} \phi^{2}(\mathbf{x}, t)+j(\mathbf{x}, t) \phi^{2}(\mathbf{x}, t) .
$$

The latter model mimics the scalar field interacting linearly with the external source

$$
\mathcal{L}=\frac{1}{2} \partial_{\mu} \phi(\mathbf{x}, t) \partial^{\mu} \phi(\mathbf{x}, t)-\frac{m_{0}^{2}}{2} \phi^{2}(\mathbf{x}, t)+j(\mathbf{x}, t) \phi(\mathbf{x}, t) .
$$

As the simplest open system we consider a time-dependent oscillator described by the single-mode Hamiltonian

$$
\hat{H}(t)=\Omega^{(\mathrm{D})}(t)\left(\hat{a}^{\dagger} \hat{a}+\frac{1}{2}\right)+\Omega^{(\mathrm{O})}(t)\left(\frac{1}{2} \hat{a}^{\dagger 2}+\frac{1}{2} \hat{a}^{2}\right),
$$

where

$$
\hat{a}=\sqrt{\frac{\omega_{0}}{2}} \hat{q}+\mathrm{i} \frac{1}{\sqrt{2 \omega_{0}}} \hat{p}, \quad \hat{a}^{\dagger}=\sqrt{\frac{\omega_{0}}{2}} \hat{q}-\mathrm{i} \frac{1}{\sqrt{2 \omega_{0}}} \hat{p} .
$$

These operators are the standard annihilation and creation operators of the timeindependent oscillator

$$
\hat{H}_{0}=\frac{1}{2} \hat{p}^{2}+\frac{\omega_{0}^{2}}{2} \hat{q}^{2} .
$$

We look for a pair of first order invariant operators of the form

$$
\left(\begin{array}{c}
\hat{A}(t) \\
\hat{A}^{\dagger}(t)
\end{array}\right)=\left(\begin{array}{cc}
u(t) & v(t) \\
v^{*}(t) & u^{*}(t)
\end{array}\right)\left(\begin{array}{c}
\hat{a} \\
\hat{a}^{\dagger}
\end{array}\right)
$$

where

$$
\mathrm{i} \frac{\mathrm{d}}{\mathrm{d} t}\left(\begin{array}{c}
u(t) \\
v(t)
\end{array}\right)+\left(\begin{array}{ll}
\Omega^{(\mathrm{D})}(t) & -\Omega^{(\mathrm{O})}(t) \\
\Omega^{(\mathrm{O})}(t) & -\Omega^{(\mathrm{D})}(t)
\end{array}\right)\left(\begin{array}{c}
u(t) \\
v(t)
\end{array}\right)=0 .
$$

And we further impose the standard commutation relation

$$
\left[\hat{A}(t), \hat{A}^{\dagger}(t)\right]=1,
$$

which leads to the unimodular matrix

$$
\mathbf{S}(t)=\left(\begin{array}{cc}
u(t) & v(t) \\
v^{*}(t) & u^{*}(t)
\end{array}\right), \operatorname{det}(\mathbf{S})=1
$$

The inverse transformation is given by

$$
\left(\begin{array}{c}
\hat{a} \\
\hat{a}^{\dagger}
\end{array}\right)=\left(\begin{array}{cc}
u^{*}(t) & -v(t) \\
-v^{*}(t) & u(t)
\end{array}\right)\left(\begin{array}{c}
\hat{A}(t) \\
\hat{A}^{\dagger}(t)
\end{array}\right) .
$$


With respect to the vacuum state (15) the position and momentum dispersion relations are calculated to be

$$
\left\langle\hat{q}^{2}\right\rangle_{(\mathrm{vac})}=\frac{1}{2 \omega_{0}}|u(t)-v(t)|^{2}, \quad\left\langle\hat{p}^{2}\right\rangle_{(\mathrm{vac})}=\frac{\omega_{0}}{2}|u(t)+v(t)|^{2},
$$

and the uncertainty relation to be

$$
\left\langle\hat{q}^{2}\right\rangle_{(\mathrm{vac})}\left\langle\hat{p}^{2}\right\rangle_{(\mathrm{vac})}=\frac{1}{4}\left\{1-\left(u(t) v^{*}(t)-u^{*}(t) v(t)\right)^{2}\right\} .
$$

By parameterizing equation (28)

$$
u(t)=\cosh r \mathrm{e}^{\mathrm{i} \theta_{u}}, \quad v(t)=\sinh r \mathrm{e}^{\mathrm{i} \theta_{v}},
$$

the uncertainty relation can be rewritten as

$$
\left\langle\hat{q}^{2}\right\rangle_{(\mathrm{vac})}\left\langle\hat{p}^{2}\right\rangle_{(\mathrm{vac})}=\frac{1}{4}\left\{1+\sinh ^{2} r \sin ^{2}\left(\theta_{u}-\theta_{v}\right)\right\} .
$$

Using the density operator (16), the thermal dispersion function is found

$$
\left\langle\hat{q}^{2}\right\rangle_{(\text {ther })}=\frac{1}{2 \omega_{0}} \operatorname{coth}\left(\frac{\beta \omega_{0}}{2}\right)|u(t)-v(t)|^{2} .
$$

The second model is a system of coupled oscillators whose coupling constants depend on time. The Hamiltonian is described by

$$
\hat{H}(t)=\sum_{\alpha \beta} \Omega_{\alpha \beta}^{(\mathrm{D})}(t)\left(\hat{a}_{\alpha}^{\dagger} \hat{a}_{\beta}+\frac{1}{2} \delta_{\alpha \beta}\right)+\Omega_{\alpha \beta}^{(\mathrm{O})}(t) \frac{1}{2} \hat{a}_{\alpha}^{\dagger} \hat{a}_{\beta}^{\dagger}+\Omega_{\alpha \beta}^{(O) *}(t) \frac{1}{2} \hat{a}_{\alpha} \hat{a}_{\beta},
$$

where $\Omega_{\alpha \beta}^{*}=\Omega_{\beta \alpha}$ to guarantee the hermiticity of the Hamiltonian. As for the first model we may find pairs of first order invariant operators

$$
\begin{aligned}
& \hat{A}_{\alpha}(t)=\sum_{\beta} u_{\alpha \beta}(t) \hat{a}_{\beta}+v_{\alpha \beta}(t) \hat{a}_{\beta}^{\dagger}, \\
& \hat{A}_{\alpha}^{\dagger}(t)=\sum_{\beta} v_{\alpha \beta}^{*}(t) \hat{a}_{\beta}+u_{\alpha \beta}^{*}(t) \hat{a}_{\beta}^{\dagger},
\end{aligned}
$$

It is straightforward to see that equation (2) leads to the system of equations

$$
\begin{aligned}
& \mathrm{i} \dot{u}_{\alpha \beta}(t)+\sum_{\gamma} \Omega_{\gamma \beta}^{(\mathrm{D})}(t) u_{\alpha \gamma}-\sum_{\gamma}\left(\Omega_{\gamma \beta}^{(O) *}(t)+\Omega_{\beta \gamma}^{(O) *}(t)\right) v_{\alpha \gamma}(t)=0, \\
& \mathrm{i} \dot{v}_{\alpha \beta}(t)-\sum_{\gamma} \Omega_{\gamma \beta}^{(\mathrm{D})}(t) v_{\alpha \gamma}+\sum_{\gamma}\left(\Omega_{\gamma \beta}^{(\mathrm{O})}(t)+\Omega_{\beta \gamma}^{(\mathrm{O})}(t)\right) u_{\alpha \gamma}(t)=0 .
\end{aligned}
$$

The commutation relations

$$
\left[\hat{A}_{\alpha}(t), \hat{A}_{\beta}^{\dagger}(t)\right]=\delta_{\alpha \beta},
$$


lead to the conditions

$$
\sum_{\gamma}\left(u_{\alpha \gamma}(t) u_{\beta \gamma}^{*}(t)-v_{\alpha \gamma}(t) v_{\beta \gamma}^{*}(t)\right)=\delta_{\alpha \beta} .
$$

The final model for the open system is a system oscillator coupled to an environment described by the Hamiltonian

$$
\hat{H}=\hat{H}_{(\text {sys })}+\hat{H}_{(\text {sys-env) }},
$$

where the system and the interaction are

$$
\begin{aligned}
\hat{H}_{(\mathrm{sys})} & =\omega_{0} \hat{a}^{\dagger} \hat{a} \\
\hat{H}_{\text {(sys-env) }} & =\nu(t) \hat{a}^{\dagger}+\nu^{*}(t) \hat{a} .
\end{aligned}
$$

Here, $\nu(t)$ is a complex function and thus of particular interest is a stochastic function. Extending the algebra by including the identity operator $\hat{e}$, a pair of first order invariant operators of the form are found

$$
\left(\begin{array}{c}
\hat{A}(t) \\
\hat{A}^{\dagger}(t)
\end{array}\right)=\left(\begin{array}{cc}
u(t) & v(t) \\
v^{*}(t) & u^{*}(t)
\end{array}\right)\left(\begin{array}{c}
\hat{a} \\
\hat{a}^{\dagger}
\end{array}\right)+\left(\begin{array}{c}
w(t) \hat{e} \\
w^{*}(t) \hat{e}
\end{array}\right)
$$

The LvN equation (2) is satisfied with the solutions

$$
\begin{aligned}
& u(t)=u_{0} \mathrm{e}^{\mathrm{i} \omega_{0} t}, \quad v(t)=v_{0} \mathrm{e}^{-\mathrm{i} \omega_{0} t}, \\
& w(t)=\mathrm{i} \int_{0}^{t} \mathrm{~d} t\left(u_{0} \nu(t) \mathrm{e}^{\mathrm{i} \omega_{0} t}-v_{0} \nu^{*}(t) \mathrm{e}^{-\mathrm{i} \omega_{0} t}\right) .
\end{aligned}
$$

The Fock space is similarly constructed as in equation (15).

Using the inverse transformation

$$
\left(\begin{array}{c}
\hat{a} \\
\hat{a}^{\dagger}
\end{array}\right)=\left(\begin{array}{cc}
u^{*}(t) & -v(t) \\
-v^{*}(t) & u(t)
\end{array}\right)\left(\begin{array}{c}
\hat{A}(t)-w(t) \hat{e} \\
\hat{A}^{\dagger}(t)-w^{*}(t) \hat{e}
\end{array}\right)
$$

it is straightforward to obtain the thermal expectation value of the system Hamiltonian

$$
\left\langle\hat{H}_{(\mathrm{sys})}\right\rangle_{(\text {ther })}=\omega_{0}\left(u_{0}^{*} u_{0}+v_{0}^{*} v_{0}\right) \frac{1}{\mathrm{e}^{\beta \omega_{0}}-1}+\omega_{0} v_{0}^{*} v_{0}+\omega_{0}\left|u(t) w^{*}(t)-v(t) w(t)\right|^{2} .
$$

The stochastic force has got the correlation functions

$$
\begin{aligned}
& \langle\nu(t)\rangle_{(\nu)}=\left\langle\nu^{*}(t)\right\rangle_{(\nu)}=0, \\
& \left\langle\nu\left(t^{\prime}\right) \nu(t)\right\rangle_{(\nu)}=\left\langle\nu^{*}\left(t^{\prime}\right) \nu^{*}(t)\right\rangle_{(\nu)}=0, \\
& \left\langle\nu^{*}\left(t^{\prime}\right) \nu(t)\right\rangle_{(\nu)}=f\left(t^{\prime}-t\right),
\end{aligned}
$$

where the average is taken with respect to a probability distribution of fluctuating parameters. For a delta-correlated force

$$
\left\langle\nu^{*}\left(t^{\prime}\right) \nu(t)\right\rangle_{(\nu)}=D \delta\left(t^{\prime}-t\right),
$$


the energy expectation value becomes

$$
\left\langle\hat{H}_{(\mathrm{sys})}\right\rangle_{(\text {ther })}=\omega_{0}\left(u_{0}^{*} u_{0}+v_{0}^{*} v_{0}\right) \frac{1}{\mathrm{e}^{\beta \omega_{0}}-1}+\omega_{0} v_{0}^{*} v_{0}+\omega_{0} D t .
$$

Thus the expectation value increases in proportion to the time of interaction with the external driving force (47). Similarly, for a Gaussian-correlated force

$$
\left\langle\nu^{*}\left(t^{\prime}\right) \nu(t)\right\rangle_{(\nu)}=D \gamma \mathrm{e}^{-\gamma\left|t^{\prime}-t\right|},
$$

we obtain the energy expectation value

$$
\begin{aligned}
\left\langle\hat{H}_{(\mathrm{sys})}\right\rangle_{\text {(ther })}= & \omega_{0}\left(u_{0}^{*} u_{0}+v_{0}^{*} v_{0}\right) \frac{1}{\mathrm{e}^{\beta \omega_{0}}-1}+\omega_{0} v_{0}^{*} v_{0}-\frac{2 D \gamma\left(\gamma^{2}-\omega_{0}^{2}\right)}{\gamma^{2}+\omega_{0}^{2}} \\
& +\frac{2 D \gamma^{2}}{\gamma^{2}+\omega_{0}^{2}} t+\frac{2 D \gamma \mathrm{e}^{-\gamma t}}{\gamma^{2}+\omega_{0}^{2}}\left\{\left(\gamma^{2}-\omega_{0}^{2}\right) \cos \omega_{0} t-2 \gamma \omega_{0} \sin \omega_{0} t\right\} .
\end{aligned}
$$

The expectation value has got constant terms, a linearly increasing term and oscillating but decaying terms.

We are able to get the well-known results for these models [1]. However, the LvN approach provides us with a clear picture for the open quantum system by making use of all the lessons from quantum mechanics and proves technically much simpler and straightforward than other methods.

\section{Open fermion system}

It was not until a quite recent time that the $\mathrm{LvN}$ approach has been applied to fermion systems [10,11]. The description of fermion systems requires not only the annihilation and the creation operators $\hat{b}, \hat{b}^{\dagger}$ for the particle but also those $\hat{d}, \hat{d}^{\dagger}$ for the antiparticle. These operators satisfy the standard anticommutation relations

$$
\begin{aligned}
& \left\{\hat{b}, \hat{b}^{\dagger}\right\}=\left\{\hat{d}, \hat{d}^{\dagger}\right\}=1, \\
& \{\hat{b}, \hat{b}\}=\left\{\hat{b}^{\dagger}, \hat{b}^{\dagger}\right\}=\{\hat{d}, \hat{d}\}=\left\{\hat{d}^{\dagger}, \hat{d}^{\dagger}\right\}=0, \\
& \{\hat{b}, \hat{d}\}=\left\{\hat{b}, \hat{d}^{\dagger}\right\}=\left\{\hat{b}^{\dagger}, \hat{d}\right\}=\left\{\hat{b}^{\dagger}, \hat{d}^{\dagger}\right\}=0 .
\end{aligned}
$$

The interaction between the fermion system and an external source requires the Grassmann variables $\hat{c}$ and $\hat{c}^{\dagger}$ that satisfy the anticommutation relations

$$
\begin{aligned}
& \left\{\hat{c}, \hat{c}^{\dagger}\right\}=1,\{\hat{c}, \hat{c}\}=\left\{\hat{c}^{\dagger}, \hat{c}^{\dagger}\right\}=0, \\
& \{\hat{c}, \hat{b}\}=\left\{\hat{c}, \hat{b}^{\dagger}\right\}=\{\hat{c}, \hat{d}\}=\left\{\hat{c}, \hat{d}^{\dagger}\right\}=0, \\
& \left\{\hat{c}^{\dagger}, \hat{b}\right\}=\left\{\hat{c}^{\dagger}, \hat{b}^{\dagger}\right\}=\left\{\hat{c}^{\dagger}, \hat{d}\right\}=\left\{\hat{c}^{\dagger}, \hat{d}^{\dagger}\right\}=0 .
\end{aligned}
$$

The first open fermion model is described by the Hamiltonian

$$
\hat{H}(t)=\hat{H}_{(\text {sys })}+\hat{H}_{(\text {sys-env) }}(t),
$$


where the system and the interaction are given by

$$
\begin{aligned}
\hat{H}_{\text {(sys) }} & =\omega_{0} \hat{b}^{\dagger} \hat{b} \\
\hat{H}_{\text {(sys-env) }}(t) & =\zeta(t) \hat{c} \hat{b}^{\dagger}+\zeta^{*}(t) \hat{b} \hat{c}^{\dagger} .
\end{aligned}
$$

This model shares all the essential characteristics of a Dirac field interacting with an external source which is described by the Lagrangian

$$
\mathcal{L}=\bar{\psi}\left(\mathrm{i} \gamma_{k} \partial_{k}+m\right) \psi+\bar{\psi} \xi(t)+\bar{\xi}(t) \psi
$$

We may find a pair of invariant operators

$$
\left(\begin{array}{c}
\hat{B}(t) \\
\hat{B}^{\dagger}(t)
\end{array}\right)=\left(\begin{array}{cc}
u(t) & v(t) \\
v^{*}(t) & u^{*}(t)
\end{array}\right)\left(\begin{array}{c}
\hat{b} \\
\hat{b}^{\dagger}
\end{array}\right)+\left(\begin{array}{c}
w^{(-)}(t) \hat{c}+w^{(+)} \hat{c}^{\dagger} \\
w^{(+) *}(t) \hat{c}+w^{(-) *} \hat{c}^{\dagger}
\end{array}\right)
$$

where

$$
\begin{aligned}
& \ddot{u}(t)-\left(\frac{\dot{\zeta}^{*}(t)}{\zeta^{*}(t)}+\mathrm{i} \omega_{0}\right) \dot{u}+\left(\zeta(t) \zeta^{*}(t)+\mathrm{i} \omega_{0} \frac{\dot{\zeta}^{*}(t)}{\zeta^{*}(t)}\right) u(t)=0, \\
& \ddot{v}(t)-\left(\frac{\dot{\zeta}(t)}{\zeta(t)}-\mathrm{i} \omega_{0}\right) \dot{v}(t)+\left(\zeta(t) \zeta^{*}(t)-\mathrm{i} \omega_{0} \frac{\dot{\zeta}(t)}{\zeta(t)}\right) v(t)=0, \\
& \mathrm{i} \dot{w}^{(-)}(t)-\zeta(t) u(t)=0, \\
& \mathrm{i} \dot{w}^{(+)}(t)+\zeta^{*}(t) v(t)=0 .
\end{aligned}
$$

Since equations (58) and (60) are the complex conjugate of equations (57) and (59), respectively, solutions can be found of the form

$$
v(t)=v_{0}^{*} u^{*}(t), w^{(+)}=v_{0}^{*} w^{(-) *}(t),
$$

where $v_{0}$ is a complex number. We further impose the anticommutation relation for all times

$$
\left\{\hat{B}(t), \hat{B}^{\dagger}(t)\right\}=1
$$

which leads to the condition

$$
\left(u^{*}(t) u(t)+w^{(-) *}(t) w^{(-)}(t)\right)\left(1+v_{0}^{*} v_{0}\right)=1 .
$$

Under the assumption that the external source provides a stochastic force with the correlations functions

$$
\langle\zeta(t) \hat{c}\rangle_{(\zeta)}=\left\langle\zeta^{*}(t) \hat{c}^{\dagger}\right\rangle_{(\zeta)}=0, \quad\left\langle\zeta\left(t^{\prime}\right) \hat{c} \zeta^{*}(t) \hat{c}^{\dagger}\right\rangle_{(\zeta)}=f\left(t^{\prime}-t\right),
$$

we find the expectation value of the Hamiltonian with respect to the initial thermal equilibrium

$$
\begin{array}{r}
\left\langle\hat{H}_{\text {(sys) }}\right\rangle_{\text {(ther) }}=\frac{1}{u^{*}(t) u(t)\left(1-v_{0}^{*} v_{0}\right)}\left[\frac{1}{\mathrm{e}^{\beta \omega_{0}}+1}+\frac{v_{0}^{*} v_{0}}{1-v_{0}^{*} v_{0}}\left(1+2 w^{(-) *}(t) w^{(-)}(t)\right)\right. \\
\left.+\int_{0}^{t} \mathrm{~d} t^{\prime} \int_{0}^{t} \mathrm{~d} t^{\prime \prime} f\left(t^{\prime}-t^{\prime \prime}\right) u^{*}\left(t^{\prime}\right) u\left(t^{\prime \prime}\right)\right] .
\end{array}
$$


The next open fermion model is motivated by a Dirac field interacting with a scalar field

$$
\mathcal{L}=\bar{\psi}\left(\mathrm{i} \gamma_{k} \partial_{k}+m\right) \psi+\phi(t) \bar{\psi} \psi
$$

Again without loss of generality we confine our attention to the single-mode quadratic Hamiltonian

$$
\hat{H}(t)=\hat{H}_{\text {(sys) }}(t)+\hat{H}_{\text {(sys-env) }}(t),
$$

where the system and the system-environment Hamiltonians are, respectively,

$$
\begin{aligned}
\hat{H}_{(\mathrm{sys})}(t) & =\Omega^{(\mathrm{D})}(t)\left(\hat{b}^{\dagger} \hat{b}-\hat{d} \hat{d}^{\dagger}\right), \\
\hat{H}_{\text {(sys-env) }}(t) & =\Omega^{\left(\mathrm{O}_{\mathrm{c}}\right)}(t) \hat{b}^{\dagger} \hat{d}^{\dagger}-\Omega^{\left(\mathrm{O}_{\mathrm{c}}\right) *}(t) \hat{b} \hat{d}+\Omega^{\left(\mathrm{O}_{\mathrm{e}}\right)}(t) \hat{b}^{\dagger} \hat{d}-\Omega^{\left(\mathrm{O}_{\mathrm{e}}\right) *}(t) \hat{b} \hat{d}^{\dagger} .
\end{aligned}
$$

We assume $\Omega^{(\mathrm{D}) *}=\Omega^{(\mathrm{D})}$, so that the Hamiltonian $(67)$ becomes the unitary operator and its evolution preserves the unitarity.

In [11] the following type of invariant operators are introduced

$$
\begin{aligned}
& \hat{B}(t)=u_{\mathrm{B}}^{-}(t) \hat{b}+u_{\mathrm{B}}^{+}(t) \hat{b}^{\dagger}+z_{\mathrm{B}}^{-}(t) \hat{d}+z_{\mathrm{B}}^{+}(t) \hat{d}^{\dagger}, \\
& \hat{D}(t)=u_{\mathrm{D}}^{-}(t) \hat{b}+u_{\mathrm{D}}^{+}(t) \hat{b}^{\dagger}+z_{\mathrm{D}}^{-}(t) \hat{d}+z_{\mathrm{D}}^{+}(t) \hat{d}^{\dagger}(t) .
\end{aligned}
$$

In fact, the algebra with the operators in equation (70) is closed under the commutation relation because of the identity between the commutator and the anticommutators

$$
\left[\hat{\mathcal{O}}_{1}, \hat{\mathcal{O}}_{2} \hat{\mathcal{O}}_{3}\right]=\left\{\hat{\mathcal{O}}_{1}, \hat{\mathcal{O}}_{2}\right\} \hat{\mathcal{O}}_{3}-\hat{\mathcal{O}}_{2}\left\{\hat{\mathcal{O}}_{1}, \hat{\mathcal{O}}_{3}\right\}
$$

The $\mathrm{LvN}$ equation leads to the following vector equation

$$
\mathrm{i} \frac{\mathrm{d}}{\mathrm{d} t}\left(\begin{array}{c}
u^{-}(t) \\
u^{+}(t) \\
z^{-}(t) \\
z^{+}(t)
\end{array}\right)+\left(\begin{array}{cccc}
\Omega^{(\mathrm{D})}(t) & 0 & \Omega^{\left(\mathrm{O}_{\mathrm{e}}\right) *}(t) & \Omega^{\left(\mathrm{O}_{\mathrm{c}}\right) *}(t) \\
0 & -\Omega^{(\mathrm{D})}(t) & -\Omega^{\left(\mathrm{O}_{\mathrm{c}}\right)}(t) & \Omega^{\left(\mathrm{O}_{\mathrm{e}}\right)}(t) \\
\Omega^{\left(\mathrm{O}_{\mathrm{e}}\right)}(t) & -\Omega^{\left(\mathrm{O}_{\mathrm{c}}\right)^{*}}(t) & \Omega^{(\mathrm{D})}(t) & 0 \\
\Omega^{\left(\mathrm{O}_{\mathrm{c}}\right)}(t) & -\Omega^{\left(\mathrm{O}_{\mathrm{e}}\right)^{*}}(t) & 0 & -\Omega^{(\mathrm{D})}(t)
\end{array}\right)\left(\begin{array}{c}
u^{-}(t) \\
u^{+}(t) \\
z^{-}(t) \\
z^{+}(t)
\end{array}\right)=0 .
$$

To simplify equation (72) two column vectors are introduced

$$
U(t)=\frac{1}{\sqrt{2}}\left(\begin{array}{c}
u^{-}(t)+u^{+}(t) \\
u^{-}(t)-u^{+}(t)
\end{array}\right), \quad Z(t)=\frac{1}{\sqrt{2}}\left(\begin{array}{c}
z^{-}(t)+z^{+}(t) \\
z^{-}(t)-z^{+}(t)
\end{array}\right),
$$

in terms of which it can be rewritten as

$$
\mathrm{i} \frac{\mathrm{d}}{\mathrm{d} t}\left(\begin{array}{c}
U(t) \\
Z(t)
\end{array}\right)+\left(\begin{array}{cc}
\Omega^{(\mathrm{D})}(t) \sigma_{1} & M_{F}(t) \\
M_{G}(t) & \Omega^{(\mathrm{D})}(t) \sigma_{1}
\end{array}\right)\left(\begin{array}{c}
U(t) \\
Z(t)
\end{array}\right)=0 .
$$

Here,

$$
\begin{aligned}
& M_{F}(t)=\Delta^{(0)}(t) I+\Delta^{(1)}(t) \sigma_{1}+\Delta^{(2)}(t) \sigma_{2}+\Delta^{(3)}(t) \sigma_{3}, \\
& M_{G}(t)=-\Delta^{(0)}(t) I+\Delta^{(1)}(t) \sigma_{1}-\Delta^{(2)}(t) \sigma_{2}-\Delta^{(3)}(t) \sigma_{3}
\end{aligned}
$$

where $\sigma_{i}$ are Pauli spin matrices and

$$
\begin{aligned}
\Delta^{(0)}(t) & =-\frac{1}{2}\left(\Omega^{\left(\mathrm{O}_{\mathrm{e}}\right)}(t)-\Omega^{\left(\mathrm{O}_{\mathrm{e}}\right) *}(t)\right), \quad \Delta^{(1)}(t)=\frac{1}{2}\left(\Omega^{\left(\mathrm{O}_{\mathrm{e}}\right)}(t)+\Omega^{\left(\mathrm{O}_{\mathrm{e}}\right) *}(t)\right), \\
\Delta^{(2)}(t) & =-\frac{\mathrm{i}}{2}\left(\Omega^{\left(\mathrm{O}_{\mathrm{c}}\right)}(t)+\Omega^{\left(\mathrm{O}_{\mathrm{c}}\right) *}(t)\right), \quad \Delta^{(3)}(t)=\frac{1}{2}\left(\Omega^{\left(\mathrm{O}_{\mathrm{c}}\right)}(t)-\Omega^{\left(\mathrm{O}_{\mathrm{c}}\right) *}(t)\right) .
\end{aligned}
$$


Further, it was shown in [11] that the anticommutation relations are consistently satisfied at all times

$$
\begin{aligned}
& \left\{\hat{B}(t), \hat{B}^{\dagger}(t)\right\}=\left\{\hat{D}(t), \hat{D}^{\dagger}(t)\right\}=1, \\
& \{\hat{B}(t), \hat{B}(t)\}=\left\{\hat{B}^{\dagger}(t), \hat{B}^{\dagger}(t)\right\}=\{\hat{D}(t), \hat{D}(t)\}=\left\{\hat{D}^{\dagger}(t), \hat{D}^{\dagger}(t)\right\}=0,
\end{aligned}
$$

Now, $\hat{B}_{\alpha}(t), \hat{B}_{\alpha}^{\dagger}(t)$ and $\hat{D}_{\alpha}(t), \hat{D}_{\alpha}^{\dagger}(t)$ play the role of the annihilation and creation operators for time-dependent fermion system. And the number operators for the particle and antiparticle are defined by

$$
\hat{N}_{\mathrm{B}}(t)=\hat{B}^{\dagger}(t) \hat{B}(t), \quad \hat{N}_{\mathrm{D}}(t)=\hat{D}^{\dagger}(t) \hat{D}(t),
$$

whose number states provide the exact quantum states according to equation (4):

$$
\hat{N}_{\mathrm{B}}(t)\left|n_{\mathrm{B}}, t\right\rangle=n_{\mathrm{B}}\left|n_{\mathrm{B}}, t\right\rangle, \quad \hat{N}_{\mathrm{D}}(t)\left|n_{\mathrm{D}}, t\right\rangle=n_{\mathrm{D}}\left|n_{\mathrm{D}}, t\right\rangle,
$$

where $n_{\mathrm{B}}, n_{\mathrm{D}}=0,1$.

\section{Conclusion}

In this paper we have applied the recently introduced Liouville-von Neumann approach to open quantum systems of bosons and fermions. It is based on physically well-grounded assumptions. Firstly, the quantum evolution of all systems, either time-independent or time-dependent, obeys the Tomonaga-Schwinger equation or the functional Schrödinger equation. Secondly, the statistical nature of systems is determined by the density operator. Technically the Liouville-von Neumann equation is solved to yield invariant operators which determine not only the Hilbert space of exact quantum states but also the density operators. Therefore, the Liouville-von Neumann approach describes the nonequilibrium quantum evolution of open systems. Moreover, the Liouville-von Neumann approach can also be applied to open fermion systems. In summary, the Liouville-von Neumann approach is a unified method that can be applied both to the equilibrium quantum evolution of time-independent systems and to the nonequilibrium quantum evolution of time-dependent boson and fermion systems.

The author would like to thank F.C. Khanna, K.-S. Soh and J.H. Yee for collaborations and useful discussions. This work was supported by KRF under Grant No. 1998-001-D00354.

\section{References}

1. Lindenberg K., West B.J. The Nonequilibrium Statistical Mechanics of Open and Closed Systems. New York, VCH Publisher, 1990.

2. Landsman N.P., van Weert Ch.G. Real- and imaginary-time field theory at finite temperature and density. // Phys. Rep., 1987, vol. 145, p. 141-249. 
3. Umezawa H. Advanced Field Theory. New York, AIP, 1993.

4. Kim S.P. Nonequilibrium self-interacting scalar fields in cosmology: the LiouvilleNeumann approach. - In: Thermal Field Dynamics and Their Applications, edited by Heinz U. (Electronic Journal, 1998; Preprint hep-ph/9811469).

5. Lewis H.R.Jr., Riesenfeld W.B. An exact quantum theory of the time-dependent harmonic oscillator and of a charged particle in a time-dependent electromagnetic field. // J. Math. Phys., 1969, vol. 10, p. 1458-1473.

6. Kim S.P. Nonequilibrium quantum scalar fields in cosmology. - In: Thermal Field Dynamics and Their Applications. Edited by Gui Y.X., Khanna F.C., and Su Z.B., Singapore, World Scientific, 1996.

7. Kim S.P. Problem of unitarity and quantum corrections in semiclassical quantum gravity. // Phys. Rev. D, 1997, vol. 55, p. 7511-7518.

8. Cho K.H., Ji J.-Y., Kim S.P., Lee C.H., Ryu J.Y. Heisenberg-approach to the quantum evolution of scalar fields in an expanding universe. // Phys. Rev. D, 1997, vol. 56, p. $4916-4921$.

9. Kim J.K., Kim S.P. One-parameter squeezed gaussian states of a time-dependent harmonic oscillator and the selection rule for vacuum states. // J. Math. Phys. A, 1999, vol. 32, p. 2711-2718.

10. Finelli F., Grrupuso A., Venturi G. Quantum fields in an expanding universe. Preprint gr-qc/9909007.

11. Kim S.P., Santana A.E., Khannna F.C. Generalized invariants and quantum evolution of open fermionic systems. Preprint hep-th/9910189.

\title{
Нерівноважна квантова еволюція відкритих систем
}

\author{
Санґ Піо Кім
}

Відділ фізики, Національний університет Кансан, Кансан 573-701, Корея

Отримано 29 листопада 1999 р.

Для опису нерівноважної квантової еволюції відкритих систем застосовується підхід Ліувіля-фон-Ноймана. Цей підхід є узагальненням методу, що був застосований як для часовонезалежних (закритих), так і для часовозалежних (відкритих) рівноважних та нерівноважних систем. Зокрема, вивчається нерівноважна квантова еволюція осциляторних моделей для відкритих бозонних та ферміонних систем.

Ключові слова: підхід Ліувіля-фон-Ноймана, інваріантний оператор, нерівноважна квантова еволюція, відкрита бозонна система, відкрита ферміонна система

PACS: 03.65. $-w, 05.30 .-d$ 